\title{
Editorial
}

\section{Acknowledgement to Reviewers of Biomolecules in 2013}

Biomolecules Editorial Office, MDPI AG, Klybeckstrasse 64, CH-4057 Basel, Switzerland

Published: 4 March 2014

The editors of Biomolecules would like to express their sincere gratitude to the following reviewers for assessing manuscripts in 2013:

\begin{tabular}{|c|c|c|}
\hline Abe, Tomoaki & Carbonetti, Nicholas & Ferreon, Allan Chris M. \\
\hline Akhtar, Kalim & Carmichael, Gordon G & Ferry, James G. \\
\hline Alberti, Simon & Carty, Michael P. & Fraaije, Marco \\
\hline An-Yuan, Guo & Chamberlain, Stormy & Fukuhara, Shigetomo \\
\hline Andrieu-Abadie, Nathalie & Chen, Chongyi & Gago, Federico \\
\hline Bahadur, Pratap & Chou, Kuochen & García-Fruitós, Elena \\
\hline Bai, Fan & Cieplak, Marek & Gerard, Nuovo \\
\hline Bakunina, Irina & Coy, Pilar & Gomes, Claudio \\
\hline Baldessari, Alicia & Crane, Edward J. & Gotor-Fernández, Vicente \\
\hline Baumgartner, Adi & Cui, Qinghua & Gotor, Vicente \\
\hline Bechini, Alessio & Curino, Alejandro C. & Greulich, Karin \\
\hline Beckham, Thomas H. & Dalmay, Tamas & Gruber, Andreas R. \\
\hline Beke-Somfai, Tamás & Das, Kalyan & Gupta, Vineet \\
\hline Benetatos, Panayotis & De Carvalho, Carla & Hagedoorn, Peter-Leon \\
\hline Benham, Adam M. & De Vries, Ronald P. & Hanefeld, Ulf \\
\hline Berhow, Mark & Devivo, Marco & Hasunuma, Tomohisa \\
\hline Billich, Andreas & Dykes, Carrie & Helbert, William \\
\hline Blinov, Nikolay & Eako, Hiroshi & Hernaiz, María J. \\
\hline Bouvet, Michael & Elez-Martínez, Pedro & Horovitz, Amnon \\
\hline Bracher, Andreas & Fabrias, Gemma & Huwiler, Andrea \\
\hline Brindley, David & Faísca, Patrícia & Imaoka, Susumu \\
\hline Brockman, Mark A. & Faller, Peter & Iourov, Ivan \\
\hline Bruni, Paola & Fang, Xianjun & Jaeger, Henry \\
\hline Cai, Mi & Faraggi, Eshel & Jiang, Xiaoxu \\
\hline Cantone, Sara & Fariselli, Piero & Jiménez-González, Concepción \\
\hline
\end{tabular}


Jin, Minghao

Johnson, Philip

Kamiya, Noriho

Kannanagattu, Prasanth

Keillor, Jeffrey

Kholodov, Yaroslav

Kim, Byung-gee

Kim, Roger

Kolkata, Iiser

Kondo, Ryuichiro

Konvalinka, Jan

Kordula, Tomasz

Kourist, Robert

Krobath, Heinrich

Kroy, Klaus

Kwawabata, Takeshi

Lairson, L.L.

Lamb, David

LaSalle, Janine

Lavandera, Ivan

Lebrilla, Carlito B

Lee, Chang-Soo

Li, Jingjing

Li, Min

Liberles, David

Lin, Peng

Litvinov, Rustem

Lopez Garcia, Isabel

Louis, J.

Lutz, Stefan

Mahajan, R. K.

Manfredi, Kirk P.

Maraia, Richard

Markus, Henke

Meng, Qin

Merrill, Alfred H.
Meyer Zu Heringdorf, Dagmar

Montclare, Jin

Mueller, Michael

Mulholland, Adrian J.

Murate, Takashi

Nagiec, Marek M.

Nakagawa, Shinichi

Natalello, Antonino

Natarajan, Viswanathan

Nevalainen, Helena

Nishihara, Shoko

Nyberg, Scott L.

Ogawa, Yuya

Ohtake, Hisao

Olivera, Ana

Olmo, M. Del

Onda, Yayoi

Palmans, Anja R. A.

Permyakov, Eugene

Pichová, Iva

Pollegioni, Loredano

Pottecher, Julien

Prasad, Kamalesh

Pruijn, Ger J. M.

Putnam, Charles W.

Ramisetty, Sreenivasa Rao

Reetz, Manfred T.

Resch, Alissa

Richard, John P.

Rios, Leonardo

Römer, Winfried

Rosa, Angelo

Rudyk, Olena

Saba, Julie D.

Saraiva, Maria Joao

Schmeck, Bernd
Schmid, Thomas

Schug, Alexander

Sharp, Josh

Song, Jinhua

Stamatis, Haralambos

Stephanie, Huang

Sue, Shih-Che

Supuran, Claudiu T

Swenson, Erik

Takabe, Kazuaki

Tavan, Paul

Tenkanen, Maija

Thomas, Christophe M.

Tortorella, Domenico

Turoverov, Konstantin

Uversky, Vladimir

Vas, Maria

Virnau, Peter

Volkman., B. F.

Waheed, Abdul

Wallin, Stefan

Wang, Qinhong

Weldon, Sinead

Wen, Fei

Winterhalter, Mathias

Witwer, Kenneth

Woodley, John M.

$\mathrm{Xu}$, Jinbo

Yamaguchi, Yoshiki

Yang, Chaoyong James

Yin, Zhaojun

$\mathrm{Yu}$, Michael S.

Yura, Kei

Zhang, Xun

Zou, Wen-Quan

(C) 2014 by the authors; licensee MDPI, Basel, Switzerland. This article is an open access article distributed under the terms and conditions of the Creative Commons Attribution license (http://creativecommons.org/licenses/by/3.0/). 